\title{
Disclosure of a stem cell phenotype in an oral squamous cell carcinoma cell line induced by BMP-4 via an epithelial-mesenchymal transition
}

\author{
BIN QIAO ${ }^{1,2}$, NEWELL W. JOHNSON ${ }^{2}$, XIAOHUA CHEN ${ }^{3}$, RUYAO LI $^{3}$, QIAN TAO $^{3}$ and JIN GAO ${ }^{1-3}$ \\ ${ }^{1}$ School of Dentistry and Oral Health, and ${ }^{2}$ Griffith Health Institute, Griffith University, \\ Qld 4215, Australia; ${ }^{3}$ Guanghua School and Hospital of Stomatology and Institute of \\ Stomatological Research, Sun Yat-sen University, Guangzhou 510055, P.R. China
}

Received March 11, 2011; Accepted April 20, 2011

DOI: 10.3892/or.2011.1299

\begin{abstract}
A small subset of cells within a malignant neoplasm, named cancer stem cells (CSCs) or tumour initiating cells, are thought to be capable of initiating the neoplasm itself, and of driving its growth and recurrence after treatment. It is unclear whether CSCs can be identified and experimentally induced within oral squamous cell carcinoma (OSCC), although this has been reported for a number of other tumour types. In this study, we aimed to determine whether BMP-4 (bone morphogenetic protein-4) could induce epithelial-mesenchymal transition (EMT) with acquisition of stem cell-like phenotypes in a cell-culture model. Furthermore, the differential expression of $\mathrm{ABCG}$, a putative CSC marker, was determined in human normal oral mucosa and OSCC tissues at mRNA and protein level. The results showed that after treatment with BMP-4, most Tca8113 cells (a human tongue OSCC cell line) changed their morphology from slabstone to spindle-shaped, and demonstrated enhanced expression of ABCG2 compared with non-treated cells. Expression of Oct- 4 was induced in cell nuclei with up-regulation of EMT markers (Snail, Slug and vimentin), and down-regulation of E-cadherin. Interestingly, the expression of hTERT, CD44 and Bmi-1 (generally accepted as markers of CSCs) were up-regulated, but this was not synchronous with the expression of EMT markers. Tumour spheres were formed after stimulation with BMP-4, with high expression of CD44 and ABCG2. In human tissues, ABCG2 was strongly expressed in OSCC, but not in normal mucosa. This study suggests that BMP-4-mediated EMT constitutes
\end{abstract}

Correspondence to: Dr Qian Tao or Dr Jin Gao, Guanghua School and Hospital of Stomatology and Institute of Stomatological Research, Sun Yat-sen University, Guangzhou 510055, P.R. China E-mail: taoqian@mail.sysu.edu.cn

E-mail: j.gao@griffith.edu.au

Key words: cancer stem cells, bone morphogenetic protein-4, epithelial-mesenchymal transition, oral squamous cell carcinoma one possible pathway for the development of CSCs in oral cancer, implying a transient therapeutic opportunity if EMT can be interrupted early in the evolution of such a neoplasm.

\section{Introduction}

Oral squamous cell carcinoma (OSCC) shares the natural history and poor outcomes with other SCC of the upper aerodigestive tract (1). Populations of cells with cancer stem cell (CSC) properties have been described in cell lines from cancers of the colon (2), lung (3) and pancreas (4). Two methods have been described recently to isolate putative CSCs from OSCC cell lines. One is to separate them into a side population (SP) by flow cytometry, based on the high expression of the putative stem cell marker ABCG2 $(5,6)$; the other attempts to use associated CSC markers, such as Bmi-1, CD44 and Oct-4, to identify CSCs microscopically $(7,8)$. Using sections of normal human skin, ABCG2 was seldom detected on presumptive epidermal stem cells (ESCs) located within the basal layer and the SP of disaggregated epidermal cells lacked stem cell features (9). It is therefore, important to reconsider whether CSCs in OSCC originate from the transformation of the healthy oral epithelial cells equivalent of ESCs or whether they can be induced within the neoplasm once malignant transformation has occurred.

Bone morphogenetic protein-4 (BMP-4) has broad functions in regulating normal tissue patterning and neoplasia (10). Overexpression of BMP-4 has been demonstrated in malignant oral epithelium compared with normal oral mucosa and benign lesions arising therefrom (11). Stromal fibroblasts cultured from head/neck cancer tissues produce factors, including BMP-4, which affect the functional properties of normal keratinocytes in culture, including promotion of epithelialmesenchymal transition (EMT)-like alterations (12).

CSCs conceptually represent key targets for therapy. However, CSCs are hard to eliminate because of their insensitivity to chemotherapeutics and their ambiguous origin. In this study, we hypothesized that the generation of CSCs in OSCC was not restricted to the transformation of stem cells within the affected, initially normal oral epithelium but could be induced within the cancer cell population itself, via the 
process of EMT. This assumption was examined by studying the effects of BMP-4 on an OSCC cell line, showing the promotion of EMT, and that the affected cells disclosed CSC properties including the development of cell spheres. ABCG2 was found to be highly expressed in primary oral cancer lesions and in metastatic neck nodes, but not in normal tissue.

\section{Materials and methods}

Cell line and cell culture. The cell line Tca8113, derived from a human squamous cell carcinoma of the tongue (13), was purchased from China Center for Type Culture Collection and maintained in RPMI-1640 medium supplemented with $10 \%$ (v/v) FCS in 25- $\mathrm{cm}^{2}$ flasks. When cells reached $50 \%$ confluence, this medium was replaced with fresh medium containing recombinant BMP-4 at $2 \mathrm{ng} / \mathrm{ml}$ (R\&D Systems, MN, USA) and cultures maintained for 1 and 2 weeks, respectively, while untreated cells were set at 0 week as controls.

Patients, specimen collection and preparation. Following approval by the University Ethics Committee and with informed consent from the patients, fresh tissues were collected from 7 patients who underwent resections of OSCC at Guanghua School of Stomatology, Sun Yat-sen University. Those samples included 2 cervical lymph nodes containing metastatic tumour from one patient. Diagnoses were confirmed from pathology reports: cases were staged according to the International Union Against Cancer rules for Head/Neck Cancer reporting (TNM classification, 1997) (14). Patients undergoing preoperative chemo/radiation therapy were excluded. Nine cases of oral mucosal tissues were harvested as controls from patients undergoing removal of impacted teeth. All specimens were collected immediately upon surgical excision and frozen in liquid nitrogen for total RNA extraction.

Total RNA extraction. The stored patient samples described above were thawed, ground and dissolved in TRIzol reagent (Invitrogen, USA), whilst Tca8113 cell cultures were harvested, before and after treatment with BMP-4, for total RNA extraction. The RNA was reverse-transcribed to cDNA using an iScript cDNA Synthesis kit (Bio-Rad, CA, USA) according to the manufacturer's instructions.

Quantitative real-time PCR ( $P P C R)$ assay for $A B C G 2$ expression. The mRNA levels of ABCG2 in both the Tca8113 cell-model and the human samples were measured by qPCR (iCycler iQ5, Bio-Rad). The primer and probe sequences used for amplification of the ABCG2 gene were: forward, 5'-GGG TAA TCC CCA GGC CTC TA-3', reverse, 5'-CCA GCT CTG TTC TGG ATT CCA-3' and probe, 5'-FAM-AGC TCA GAT CAT TGT CAC AGT CGT ACT-TAMRA-3'; for $\beta$-actin, forward, 5'-GCA TGG GTC AGA AGG ATT CCT-3', reverse, 5'-TCG TCC CAG TTG GTG ACG AT-3', and probe, 5'-FAMCCT CAC CCT GAA GTA CCC CAT CGA GC-TAMRA-3'. Levels of the different mRNAs were subsequently normalized against $\beta$-actin mRNA levels. All experiments were performed at least in duplicate.

In order to validate the mRNA expression, we performed immunohistochemisty of ABCG2 (1:20, Boster, China). Ten additional archival paraffin-embedded specimens of normal mucosa and OSCCs each were used from the storage of the Department of Oral Pathology.

Tumour sphere assay. After treatment with BMP-4, single cells of Tca8113 were plated in ultralow attachment plates (Corning, NY, USA) at a density of 20,000 viable cells $/ \mathrm{ml}$ of primary culture and 1,000 cells $/ \mathrm{ml}$ in passages. Cells were grown in serum-free epithelial growth medium supplemented with B27 (Invitrogen) containing $20 \mathrm{ng} / \mathrm{ml}$ of epidermal growth factor and basic fibroblast growth factor (BD Biosciences, MD, USA). Tumour spheres were collected by gentle centrifugation (800 rpm) after 6 days, resuspended in PBS and cytospun onto slides for immunofluorescent double-staining of CD44 (1:100, Cell Signaling Technology, CA, USA) and ABCG2 (1:100).

Immunocytochemistry. Following treatment with BMP-4, Tca8113 cells in 96-well plates were fixed in 4\% paraformaldehyde for $10 \mathrm{~min}$ and washed with PBS. Cell membranes were permeablised with $0.2 \%$ Triton X-100/PBS for $10 \mathrm{~min}$ prior to the performance of immunocytochemistry. Primary anti-pan-cytokeratin antibody (1:100, Boster) and antivimentin $\left(1: 100\right.$, Boster) were incubated with cells at $4^{\circ} \mathrm{C}$ overnight, while immunofluorescence was used to trace the subcellular location of Oct-4 (1:100, Santa Cruz, Biotechnology, Santa Cruz, CA, USA). Non-immune serum of the same species was used as a negative control.

In vitro wound healing assay. Tca8113 cells were seeded in 6 -well culture dishes at $3 \times 10^{5}$ cells/well and incubated until confluent. An artificial wound was incised with a pipette tip in the central area of the confluent culture on the dish. Detached cells were removed carefully with PBS. BMP-4 $(5 \mathrm{ng} / \mathrm{ml})$ and serum-free medium were respectively added to each well for $48 \mathrm{~h}$ and cells which migrated into wound areas were observed by inverted microscopy every $12 \mathrm{~h}$ up to $48 \mathrm{~h}$.

Semi-quantitative reverse transcription-PCR (RT-PCR). The cDNA products gained from Tca8113 cells were assessed by conventional RT-PCR for the expression of both EMT- and CSC-related genes: the former genes included, Snail, Slug and E-cadherin; and the latter, hTERT, CD44 and Bmi-1. The primers used have been previously reported (15-18). The PCR products were resolved by electrophoresis on $2 \%$ agarose gels and visualized by ethidium bromide staining. The bands of EMT and CSC markers were normalized against the intensity of the house-keeping gene $\beta$-actin, and analysed by densitometry.

Western blots. Total protein was extracted using a standard method. Briefly, $40 \mu \mathrm{g}$ of protein of each sample was subjected to SDS-PAGE on $10 \%$ gels. Bands were transferred to PVDF membranes and blocked with $5 \%(\mathrm{w} / \mathrm{v})$ non-fat dry milk in Tris-buffered saline (TBS) for $2 \mathrm{~h}$ at room temperature. Membranes were then incubated with each primary antibody overnight at $4^{\circ} \mathrm{C}$. Antibodies used were $\alpha$-tubulin $(1: 3,000$, Abcam, USA), Slug (1:1,000, Cell Signaling Technology), Snail (1:200, Santa Cruz Biotechnology), E-cadherin (1:1,000, Invitrogen), hTERT (1:200, Boster), CD44 (1:500) and Bmi-1 (1:300, Cell Signaling Technology). Membranes were washed twice and incubated with horseradish peroxidase-conjugated secondary antibody for $2 \mathrm{~h}$ at room temperature. Subsequently, 
A

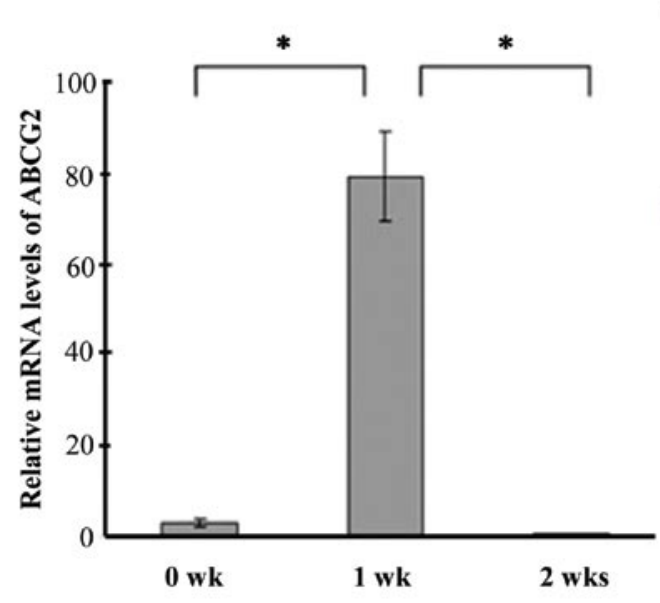

C
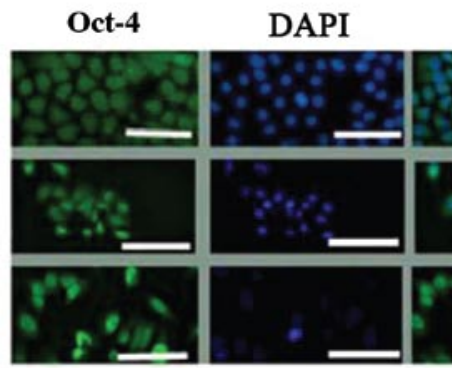

B

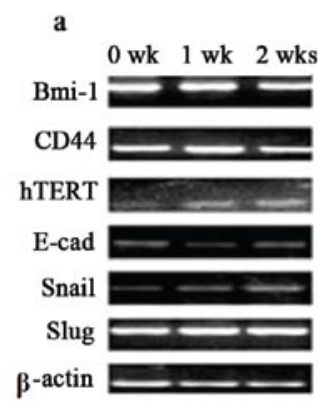

c

0 wk 1 wk 2 wks

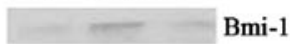

$\because \mathrm{CD} 44$

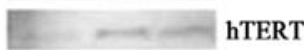

- ....... E-cad

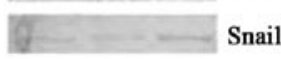

$\longrightarrow$ Slug

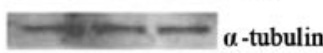

b

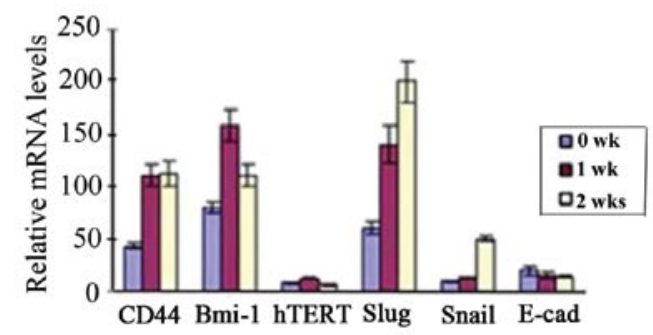

D

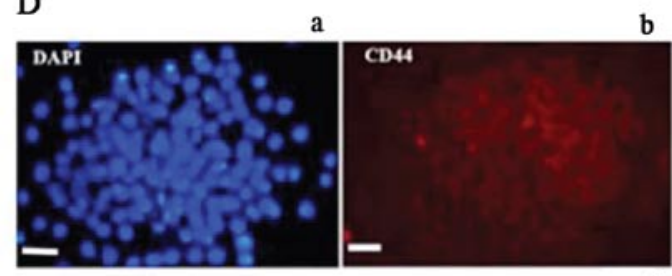

0 wk
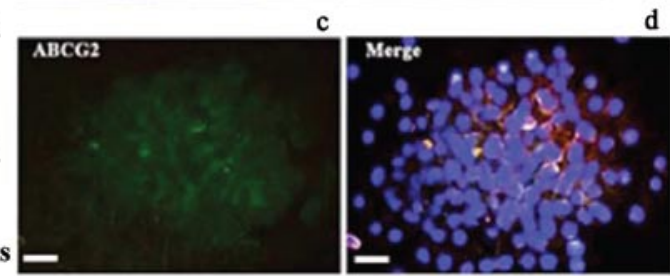

Figure 1. Enrichment of cancer stem cell properties in Tca8113 cells. (A) The mRNA of ABCG2 is transiently expressed in Tca8113 cells by real-time PCR at different time points after treatment of BMP-4. "Significantly different from the untreated and the treated for 2 weeks sample with $\mathrm{P}<0.05$. (B) Expression of a range of EMT (E-cadherin, Snail, Slug) and stem cell markers (Bmi-1, CD44, hTERT) is detected at the mRNA and protein level by RT-PCR (a) visualized bands; (b) converted to quantitative values using densitometry and (c) Western blots. (C) Oct- 4 translocation from the cytoplasm to the cell nuclei of Tca8113 cells. Bar, $50 \mu \mathrm{m}$. (D) Illustration of a typical tumour sphere. Immunofluorescent expression of CD44 (b) and ABCG2 (c), with the specific DNA stain, DAPI at (a), and a merged image in (d) (Bar, $25 \mu \mathrm{m})$.

the protein bands were detected by enhanced chemiluminescence and visualised by VersaDoc MP Imaging Systems (Bio-Rad).

Statistical analysis. Data from qPCR assays were analyzed by one-way analysis of variance, using the Statistical Analysis System 8.0. A P-value of $<0.05$ was taken to denote statistical significance.

\section{Results}

Stem cell-like phenotypes are disclosed in BMP-4-treated Tca8113 cells. We found that the mRNA expression for ABCG2 was increased dramatically in treated cells at week 1 compared with the non-treated cells, but declined at week 2 (Fig. 1A). The associated markers of EMT and of CSC were all slightly up-regulated while E-cadherin was down-regulated at teh mRNA level as determined using RT-PCR (Fig. 1Ba). Western blot analysis showed the protein expression to be consistent with the mRNA (Fig. 1Bc) and with a time-related pattern similar to that of ABCG2, with the peak expression at week 1 . Specific changes were noted in Oct-4 expression, with the product apparently moving from the cytoplasm to the nucleus (Fig. 1C) in 2-dimensional culture. In the anchorageindependent cultures, formation of tumour spheres was observed, along with high expression of CD44 and ABCG2 by immunofluorescence staining (Fig. 1D).

Snail family members are pivotal regulators of the EMT process mediated by BMP-4. Snail and Slug were up-regulated at both the mRNA and protein levels (Fig. 1B). The intensity of Snail up-regulation was not as strong at the protein level as 

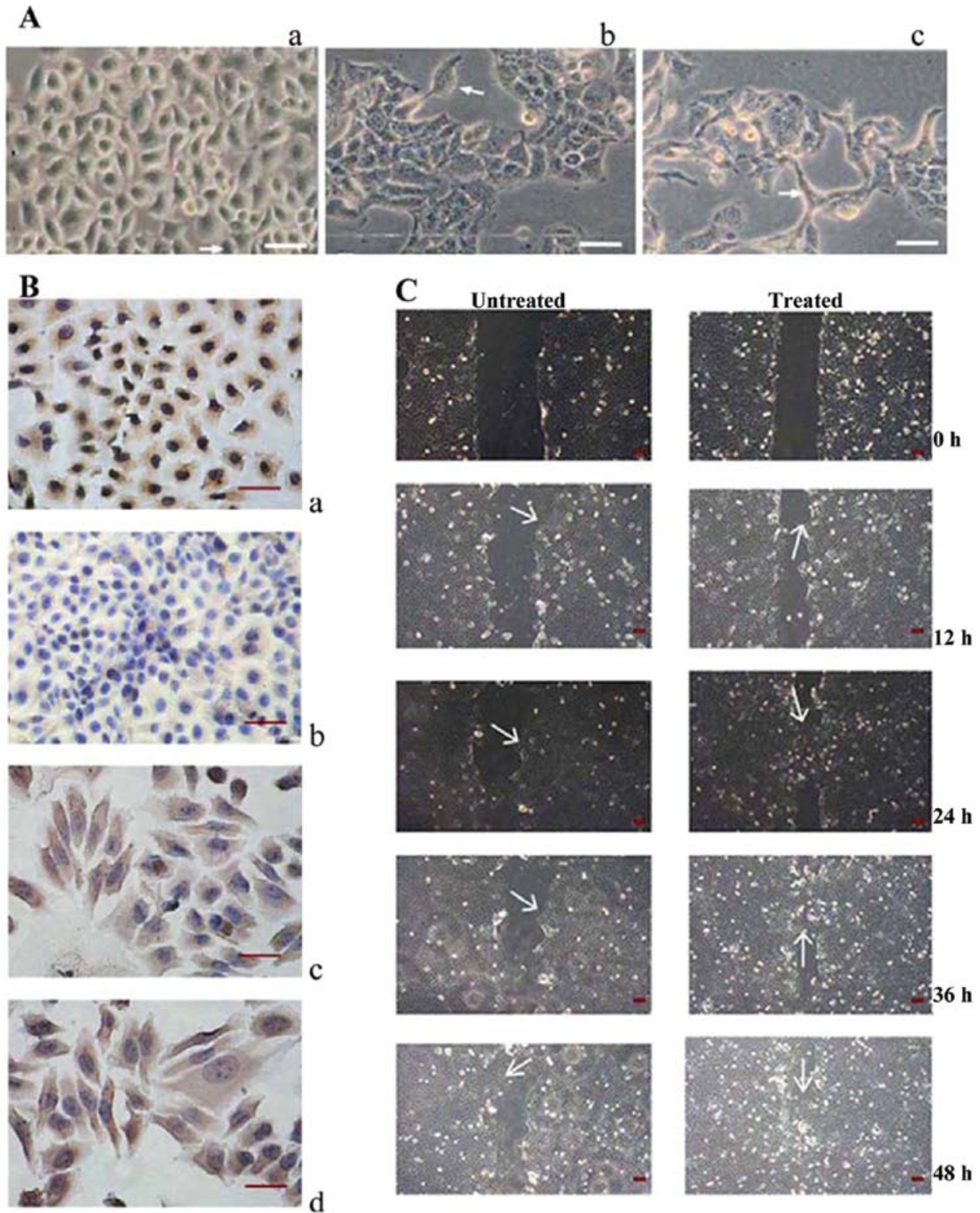

Figure 2. EMT characteristics in Tca8113 cells. (A) Cell morphology changes from slabstone (arrows) to spindle-shaped (arrows) following treatment with BMP-4: week 0 (a), week 1 (b) and week 2 (c) (Bar, $50 \mu \mathrm{m})$. (B) Immunocytochemical staining demonstrates the expression of cytokeratin (a), but not vimentin (b) in untreated Tca8113 cells; positive cytokeratin (c) and vimentin (d) staining in Tca8113 cells treated with BMP-4 for 2 weeks (Bar, $50 \mu$ m). (C) Wound healing assay. Spindle-shaped cells (arrows) appear on the margin of the artificial wounds. The speed of healing was slightly faster in BMP-4 treated cells than that in untreated cells (Bar, $150 \mu \mathrm{m}$ ).

at the mRNA level. The expression of Slug was equally clear in both assays. Such observations may indicate that Slug was the major regulator within the Snail family in Tca8113 cells.

BMP-4 mediates acquisition of mesenchymal characteristics in Tca8113 cells. During cultivation with BMP-4, cancer cells gradually changed their morphology from slabstone to spindle-shaped, particularly at week 2 (Fig. 2A). Immunocytochemistry demonstrated the presence of cytokeratin in both treated and untreated cells, but vimentin was only demonstrated in BMP-4 treated cells (Fig. 2B). Interestingly, the wound healing assay demonstrated short-term EMT as spindle-like cells appeared at the wound margin of both BMP-treated and control groups. Cells treated with BMP-4 grew slightly faster than controls (Fig. 2C).
Expression of ABCG2 varies with the nature of OSCC tissues. To help understand the significance of the expression of ABCG2 in the in vitro cell-model, clinical samples were examined by qPCR and by immunohistochemistry. Of the fresh cancer specimens, five were $\mathrm{T}_{1} \mathrm{~N}_{0} \mathrm{M}_{0}$ lesions, one $\mathrm{T}_{2} \mathrm{~N}_{0} \mathrm{M}_{0}$ and one a $\mathrm{T}_{2} \mathrm{~N}_{2} \mathrm{M}_{0}$ lesion (Fig. 3A). ABCG2 mRNA was highly expressed in all 9 primary lesions and in $2 / 2$ metastatic lymph nodes examined (Fig. 3B). No expression was detectable in the normal tissues. ABCG2 was about 2-fold higher in the lower deep cervical node than that in the upper deep cervical node (data not shown). By immunohistochemistry, ABCG2 appeared negative in the normal mucosa compared with the positive controls (Fig. 4A and B). Interestingly, the staining gradually increased from weak expression on the basement membrane of epithelial pegs (Fig. 4C and D) to infiltrating cancer nests, 
A

$\left.\begin{array}{lc}\hline \multicolumn{1}{c}{\text { Tissues }} & \text { TNM classification } \\ \hline \text { 9 normal oral mucosa } & \text { Not applicable } \\ \text { SCC of gingiva } & \mathrm{T}_{1} \mathrm{~N}_{0} \mathrm{M}_{0} \\ \text { SCC of oropharyngeal } & \mathrm{T}_{1} \mathrm{~N}_{0} \mathrm{M}_{0} \\ \text { SCC of floor of mouth } & \mathrm{T}_{1} \mathrm{~N}_{0} \mathrm{M}_{0} \\ \text { SCC of buccal mucosa } & \mathrm{T}_{1} \mathrm{~N}_{0} \mathrm{M}_{0} \\ \text { SCC of tongue } & \mathrm{T}_{1} \mathrm{~N}_{0} \mathrm{M}_{0} \\ \text { SCC of tongue } & \mathrm{T}_{2} \mathrm{~N}_{0} \mathrm{M}_{0} \\ \text { SCC of tongue } & \\ \text { Upper deep cervical lymph } & \\ \text { nodes } & \\ \text { Lower deep cervical lymph } \\ \text { nodes }\end{array}\right\}$

B

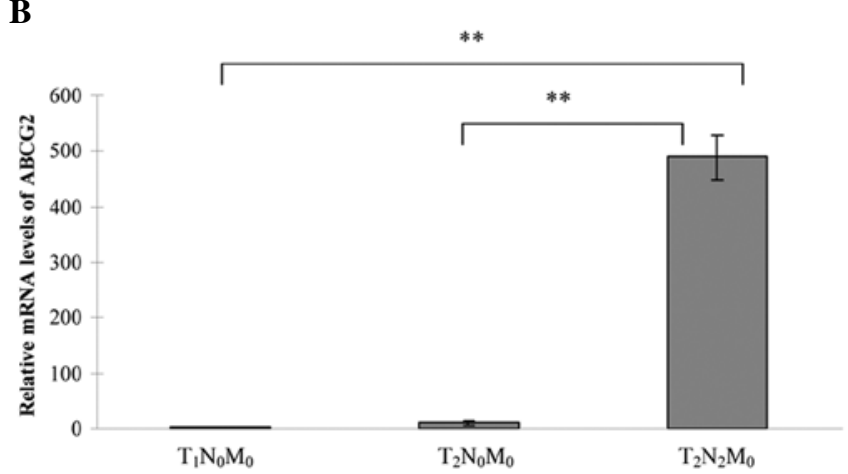

Figure 3. The mRNA expression of ABCG2 in normal oral mucosa and OSCCs using real-time PCR. (A) The locations of OSCC and TNM grade of specimen. (B) Statistical analysis of ABCG2 mRNA expression in OSCC tissues at stages $T_{1} N_{0} M_{0}, T_{2} N_{0} M_{0}$ and $T_{2} N_{2} M_{0}$. There was no expression of ABCG2 in normal oral mucosa (data not shown), while ABCG2 mRNA increased dramatically from $\mathrm{T}_{1} \mathrm{~N}_{0} \mathrm{M}_{0}$ to $\mathrm{T}_{2} \mathrm{~N}_{2} \mathrm{M}_{0} .{ }^{* *}$ Significant at $\mathrm{P}<0.01$.

particularly in lymph nodes (Fig. 4E and $\mathrm{F}$ and Table I). Furthermore, spindle-shaped cancer cells showed expression of both cytokeratin and ABCG2 in metastatic deposits (Fig. 4G and $\mathrm{H}$ ), consistent with findings from our in vitro model.

\section{Discussion}

There is considerable interest in the formation and behavior of putative CSCs driving tumour proliferation and recurrence.
However, the origin of CSCs is still unclear; some studies indicate that their origin is from the stem cells of adult selfrenewing tissues, including blood, secretory organs and stratified squamous epithelia $(19,20)$. On the other hand, Weinberg's group has demonstrated that CSCs can be enriched within an existing malignancy, by 'dedifferentiation' of mature tumor cells through an EMT pathway (21). Current cancer chemoprophylaxis focuses on the former strategy: prevention of the malignant transformation of adult stem cells to CSCs, but this generally fails. Since CSCs may be temporarily 'stored' in a state of quiescence or kept in the cell cycle at $\mathrm{G}_{0} / \mathrm{G}_{1}$ phase, this type of cell is able to escape the effects of chemopreventative or chemotherapeutic agents. This forces us to explore other potential therapeutic windows by increasing our understanding of the processes of dedifferentiation within a population of cells comprising an existing malignant neoplasm.

Using an in vitro cell-model, Tca8113 tongue cancer cells were treated with BMP-4 to determine whether EMT was involved in CSC formation. As expected, we found that cytokeratin expression remained, with decreased expression of E-cadherin, while vimentin appeared, indicating the induction of EMT. According to the literature, EMT is a transient process during tumour initiation and early growth: this is followed by mesenchymal-epithelial transition (MET), the next half circle of tumour growth (22). Significantly, in our wound healing assay, cells in the control group also underwent EMT, perhaps indicating that EMT is a normal physiological process during tissue development, remodeling and repair (23). Indeed 3 subtypes of EMT have been described (24); Type 1 EMT, which occurs during embryogenesis and organ development; Type 2 EMT, which is biophysically associated with tissue regeneration and organ fibrosis; Type 3 EMT, which is associated with invasion by neoplastic cells, and ultimately metastasis. Whilst the cells in our wound healing assay might be considered to be behaving like the Type 2 cells, given that they are an immortalized line derived from a human tongue cancer, and that EMT is readily enhanced by BMP-4, it is reasonable to assume they represent Type 3 .

In the study of EMT pathways, the Snail family of zincfinger transcription factors is generally regarded as a key player. Members of this family act upstream of E-cadherin in the control of cell adhesion and migration (25), and it is known that the TGF- $\beta$ superfamily (including BMP-4) affects Snail expression (26). Thus, in our experiments, we suggest that there is a signaling pathway whereby BMP-4 controls EMT via

Table I. The semi-quantitative analysis of immunohistochemical expression of ABCG2 in paraffin tissue sections of normal oral mucosa and OSCCs.

Tissues

Cytokeratin

ABCG2

Normal oral mucosal epithelium

Primary lesions (adjacent normal epithelial peg)

$\begin{array}{cc}+++ & -/+ \\ +++ & + \\ ++++ & ++ \\ ++++ & +++\end{array}$

Primary lesions (epithelial cancer nests)

$+++$

The expression of cytokeratin in the normal oral mucosa was set as being +++ . For ABCG $2,+$, positive expression; -/+, either negative or very weak staining. 

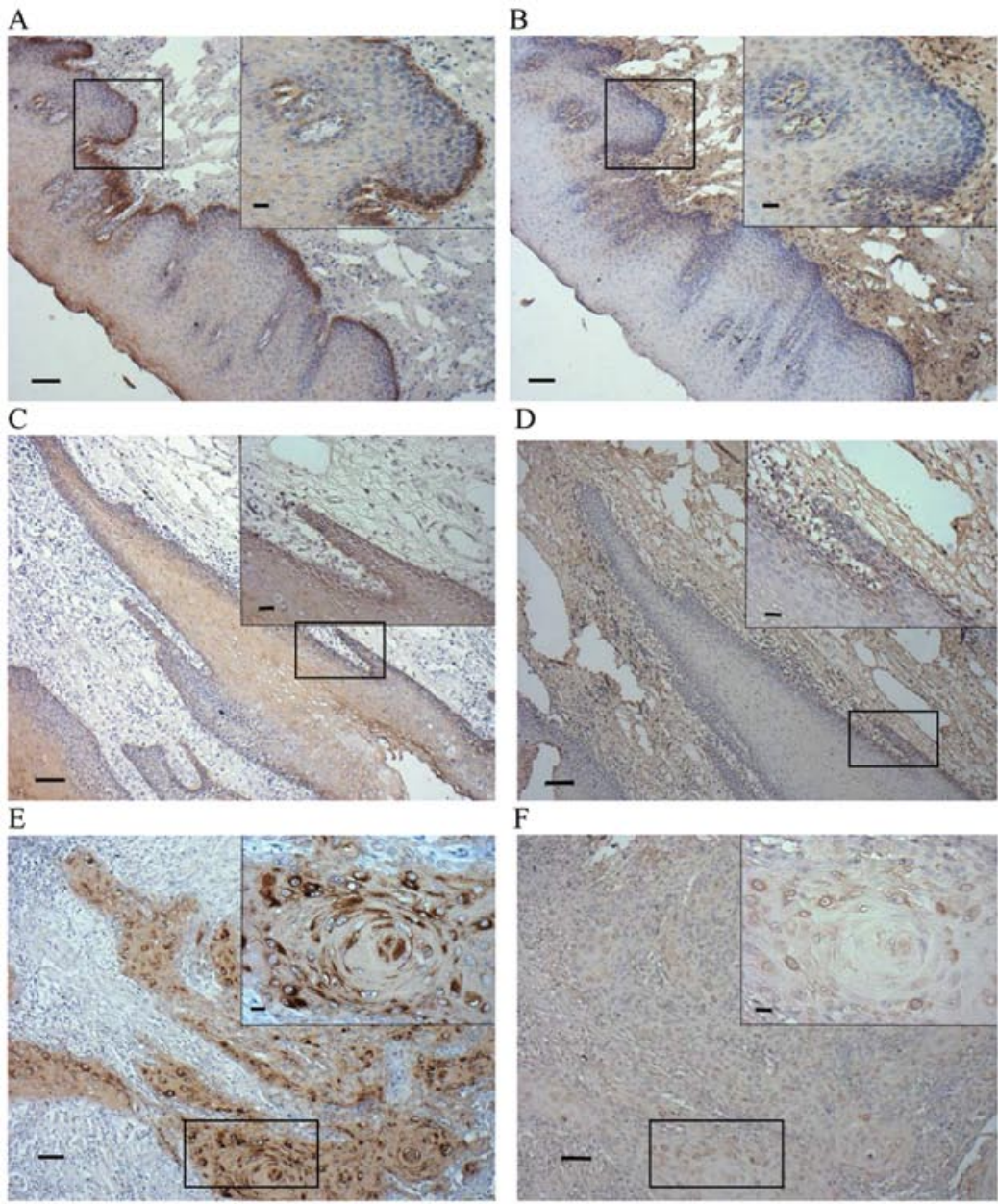

$\mathrm{F}$

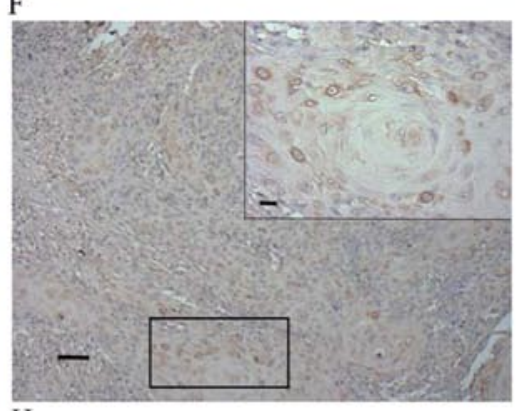

$\mathrm{G}$

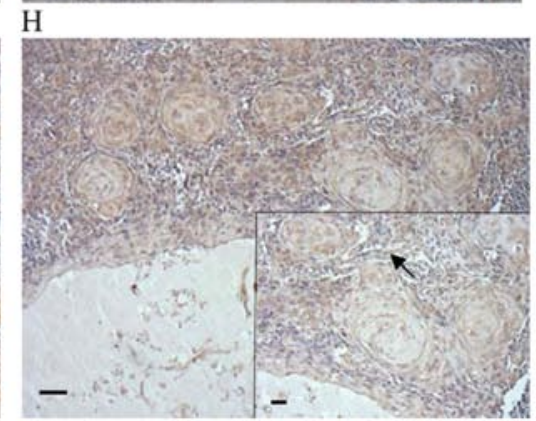

Figure 4. Representative samples of the expression of ABCG2 at the protein level in normal oral mucosa and OSCCs using immunohistochemistry (Bar, $100 \mu \mathrm{m}$ ). Cytokeratin is used as a positive control in the left panels (A, C, E and G) and ABCG2 is shown in the right panels (B, D, F and H). (A and B) normal oral mucosa; (C and D) proliferative epithelial pegs in the adjacent normal epithelium, $(\mathrm{E}$ and $\mathrm{F})$ invasive cancer cells of a primary OSCC; $(\mathrm{G}$ and $\mathrm{H})$ metastatic deposits. The inset of (B) shows the absence of ABCG2 expression in the normal epithelium, but distinct expression in invading keratinocytes are observed in (D) and (F) (Bar, $25 \mu \mathrm{m})$. The insets of (G) and (H) (Bar, $50 \mu \mathrm{m}$ ) show spindle-shaped cells (arrows) strongly positive for cytokeratin and ABCG2, respectively, in lymphatic deposits.

Snail family activity. In addition, in our studies, the expression levels of hTERT, CD44 and Bmi-1 were also increased. Importantly, the formation of tumour spheres and the translocation of Oct- 4 from the cytoplasm to the nucleus, implies that the ability for self-renewal was enhanced in our Tca 8113 cells, as also shown by Okamoto et al (7).

More importantly, the peak of ABCG2 mRNA expression in treated-Tca8113 cells was at week 1, declining unexpectedly at 2 weeks. Currently there is controversy regarding the role of ABCG2 in the process of EMT. Some studies have demonstrated an increase in ABCG2 associated with EMT (27), while others, using ABCG2 to define a side population in studies of a breast cancer cell line in vitro have shown that the SP was depleted when provoked by TGF- $\beta 1$ (28). In our study, not only was there fluctuation in $\mathrm{ABCG} 2$, but also the expression of CD44, hTERT and Bmi-1 tended to rise and fall as the culture time increased. It appears that the development of markers of EMT and of stemness may not be synchronous. Although both phenomena might be necessary for cells to acquire enhanced motility and the ability for self-renewal, fundamental prerequisites for colonisation of secondary sites (29), we suggest that the disclosure of stemness takes place at the early stage of EMT, while cell metastasis occurs at later stages of EMT. This is supported by the findings of ABCG2 
immunohistochemistry in the present study: ABCG2 was highly expressed in nests of invading malignant keratinocytes of primary lesions ahead of the appearance of spindle-shaped cells in metastatic sites. This implies a transient opportunity for elimination of CSCs before the migration of malignant cells to secondary sites.

In summary, our results indicate that the stemness of OSCC cells can be disclosed via an EMT pathway in the presence of BMP-4 stimulation. This suggests that the origin of CSCs is multiple and not restricted to transformation of adult stem cells, implying a transient therapeutic opportunity if the process of EMT can be interrupted.

\section{Acknowledgements}

This study was supported by Research Funds from both the Griffith University and the China Scholarship Council (2008638009).

\section{References}

1. McDowell JD: An overview of epidemiology and common risk factors for oral squamous cell carcinoma. Otolaryngol Clin North Am 39: 277-294, 2006.

2. O'Brien CA, Pollett A, Gallinger S and Dick JE: A human colon cancer cell capable of initiating tumour growth in immunodeficient mice. Nature 445: 106-110, 2007.

3. Eramo A, Lotti F, Sette G, Pilozzi E, Biffoni M, Di Virgilio A, Conticello C, Ruco L, Peschle C and De Maria R: Identification and expansion of the tumorigenic lung cancer stem cell population. Cell Death Differ 15: 504-514, 2008.

4. Li C, Lee CJ and Simeone DM: Identification of pancreatic cancer stem cells. Methods Mol Biol 568: 161-173, 2009.

5. Harper LJ, Piper K, Common J, Fortune F and Mackenzie IC: Stem cell patterns in cell lines derived from head and neck squamous cell carcinoma. J Oral Pathol Med 36: 594-603, 2007.

6. Zhang P, Zhang Y, Mao L, Zhang Z and Chen W: Side population in oral squamous cell carcinoma possesses tumor stem cell phenotypes. Cancer Lett 277: 227-234, 2009.

7. Okamoto A, Chikamatsu K, Sakakura K, Hatsushika K, Takahashi G and Masuyama K: Expansion and characterization of cancer stem-like cells in squamous cell carcinoma of the head and neck. Oral Oncol 45: 633-639, 2009.

8. Prince ME, Sivanandan R, Kaczorowski A, Wolf GT, Kaplan MJ, Dalerba P, Weissman IL, Clarke MF and Ailles LE: Identification of a subpopulation of cells with cancer stem cell properties in head and neck squamous cell carcinoma. Proc Natl Acad Sci USA 104: 973-978, 2007.

9. Triel C, Vestergaard ME, Bolund L, Jensen TG and Jensen UB: Side population cells in human and mouse epidermis lack stem cell characteristics. Exp Cell Res 295: 79-90, 2004.

10. Hamada S, Satoh K, Hirota M, Kimura K, Kanno A, Masamune A and Shimosegawa T: Bone morphogenetic protein 4 induces epithelial-mesenchymal transition through MSX2 induction on pancreatic cancer cell line. J Cell Physiol 213: 768-774, 2007.

11. Jin Y, Tipoe GL, Liong EC, Lau TY, Fung PC and Leung KM: Overexpression of BMP-2/4, -5 and BMPR-IA associated with malignancy of oral epithelium. Oral Oncol 37: 225-233, 2001.

12. Strnad H, Lacina L, Kolár M, Cada Z, Vlcek C, Dvoránková B, Betka J, Plzák J, Chovanec M, Sáchová J, Valach J, Urbanová M and Smetana K Jr: Head and neck squamous cancer stromal fibroblasts produce growth factors influencing phenotype of normal human keratinocytes. Histochem Cell Biol 133: 201-211, 2010.
13. Duan L, Yao J, Wu X and Fan M: Growth suppression induced by Notch1 activation involves Wnt-beta-catenin down-regulation in human tongue carcinoma cells. Biol Cell 98: 479-490, 2006.

14. Numata T, Muto H, Shiba K, Nagata H, Terada N and Konno A: Evaluation of the validity of the 1997 International Union Against Cancer TNM classification of major salivary gland carcinoma. Cancer 89: 1664-1669, 2000.

15. Eastham AM, Spencer H, Soncin F, Ritson S, Merry CL, Stern PL and Ward CM: Epithelial-mesenchymal transition events during human embryonic stem cell differentiation. Cancer Res 67: 11254-11262, 2007.

16. Boldrup L, Coates PJ, Gu X and Nylander K: DeltaNp63 isoforms regulate CD44 and keratins 4, 6, 14 and 19 in squamous cell carcinoma of head and neck. J Pathol 213: 384-391, 2007.

17. Kim JH, Yoon SY, Jeong SH, Kim SY, Moon SK, Joo JH, Lee Y, Choe IS and Kim JW: Overexpression of Bmi-1 oncoprotein correlates with axillary lymph node metastases in invasive ductal breast cancer. Breast 13: 383-388, 2004.

18. He YL, Wu YH, He XN, Liu FJ, He XY and Zhang Y: An immortalized goat mammary epithelial cell line induced with human telomerase reverse transcriptase (hTERT) gene transfer. Theriogenology 71: 1417-1424, 2009.

19. Mimeault $M$ and Batra SK: Recent advances on skin-resident stem/progenitor cell functions in skin regeneration, aging and cancers and novel anti-aging and cancer therapies. J Cell Mol Med 14: 116-134, 2010.

20. Mimeault M and Batra SK: Recent progress on normal and malignant pancreatic stem/progenitor cell research: therapeutic implications for the treatment of type 1 or 2 diabetes mellitus and aggressive pancreatic cancer. Gut 57: 1456-1468, 2008.

21. Mani SA, Guo W, Liao MJ, Eaton EN, Ayyanan A, Zhou AY, Brooks M, Reinhard F, Zhang CC, Shipitsin M, Campbell LL, Polyak K, Brisken C, Yang J and Weinberg RA: The epithelialmesenchymal transition generates cells with properties of stem cells. Cell 133: 704-715, 2008.

22. Polyak K and Weinberg RA: Transitions between epithelial and mesenchymal states: acquisition of malignant and stem cell traits. Nat Rev Cancer 9: 265-273, 2009.

23. Klymkowsky MW and Savagner P: Epithelial-mesenchymal transition: a cancer researcher's conceptual friend and foe. Am J Pathol 174: 1588-1593, 2009.

24. Kalluri R and Weinberg RA: The basics of epithelial-mesenchymal transition. J Clin Invest 119: 1420-1428, 2009.

25. Moustakas A and Heldin $\mathrm{CH}$ : Signaling networks guiding epithelial-mesenchymal transitions during embryogenesis and cancer progression. Cancer Sci 98: 1512-1520, 2007.

26. Thériault BL, Shepherd TG, Mujoomdar ML and Nachtigal MW: BMP4 induces EMT and Rho GTPase activation in human ovarian cancer cells. Carcinogenesis 28: 1153-1162, 2007.

27. Meyer zu Schwabedissen HE, Grube M, Dreisbach A, Jedlitschky G, Meissner K, Linnemann K, Fusch C, Ritter CA, Völker U and Kroemer HK: Epidermal growth factor-mediated activation of the map kinase cascade results in altered expression and function of ABCG2 (BCRP). Drug Metab Dispos 34: 524-533, 2006.

28. Yin L, Castagnino P and Assoian RK: ABCG2 expression and side population abundance regulated by a transforming growth factor beta-directed epithelial-mesenchymal transition. Cancer Res 68: 800-807, 2008.

29. Visvader JE and Lindeman GJ: Cancer stem cells in solid tumours: accumulating evidence and unresolved questions. Nat Rev Cancer 8: 755-768, 2008. 